\title{
LeGes
}

Emanuela Chiapparini

\section{Armutsbetroffene Personen evaluieren Projekte der Armutspolitik}

\section{Chancen und Voraussetzungen partizipativer Evaluation}

Die Mitwirkung von armutsbetroffenen und -gefährdeten Personen an Massnahmen und Projekten der Armutspolitik und derer Evaluation ist wirkungsvoll. Hierzu werden ein differenziertes Verständnis von Armut und Partizipation sowie geeignete Evaluationsinstrumente und Indizien zur Messung des Wirkungspotenzials vorausgesetzt. Dies wird im Beitrag basierend auf zwei partizipativen Evaluationsprojekten in der Schweiz skizziert. Zudem werden Chancen und Voraussetzungen der Evaluation mit Einbezug von armutsbetroffenen und -gefährdeten Personen herausgearbeitet.

Beitragsart: Wissenschaftliche Beiträge des SEVAL-Kongresses

Zitiervorschlag: Emanuela Chiapparini, Armutsbetroffene Personen evaluieren Projekte der Armutspolitik, in: LeGes 32 (2021) 1 


\section{Inhaltsübersicht}

I. Ausgangslage

1. Überblick

2. Armut

3. Zentrale Dimensionen der Partizipation

4. Partizipation in sechs Politik- und Handlungsbereichen der Armutsprävention und -bekämpfung

II. Partizipative Evaluationsprojekte

1. Partizipative Evaluation

2. Begleitgruppe als kollaborative partizipative Evaluation

3. Indikatorengewinnung durch evaluatorgesteuerte partizipative Evaluation (PartSoz)

III. Chancen und Voraussetzungen

IV. Geeignete Evaluationsinstrumente und Indizien für die Messung von Wirkungspotenzial

\section{Ausgangslage}

\section{1. Überblick}

[1] Verschiedene Personen beschäftigen sich mit Entscheiden und Angelegenheiten, die das Leben von armutsbetroffenen und -gefährdeten Personen betreffen: Regierungsmitglieder und kantonale Parlamente beschliessen Massnahmen und erlassen Sozialhilfegesetze. Von statistischen Ämtern werden Zahlen über das Ausmass und die Entwicklung von Armut publiziert. Sozialdiensten kommt eine unterstützende und beratende Funktion zu, und an Hochschulen werden Sozialarbeitende ausgebildet und vielfältige Forschungsbefunde zum Thema Armut gewonnen. In der Medienlandschaft und der Öffentlichkeit wird unterschiedlich differenziert über die Sozialhilfe und über armutsbetroffene Personen diskutiert. Allerdings bleibt unklar, welche Rolle armutsbetroffene Personen selbst übernehmen, wenn es um Entscheide und Angelegenheiten geht, die ihr eigenes Leben betreffen.

[2] In einem Forschungsauftrag des Bundesamts für Sozialversicherungen (BSV) untersuchen Forschende der Berner Fachhochschule (BFH) in Zusammenarbeit mit den Fachhochschulen Westschweiz Freiburg (HES-SO/FR) und Genf (HESO-SO/GE) bestehende Möglichkeiten für betroffene Personen, an Massnahmen der Armutsprävention oder -bekämpfung innerhalb und ausserhalb der Schweiz zu partizipieren. Hierzu zählt ebenfalls die Mitwirkung an der Evaluation solcher Projekte, worauf die Ausführungen dieses Beitrages fokussiert sind.

[3] Der Beitrag startet mit einer differenzierten Klärung des Armuts- und des Partizipationsbegriffs sowie mit einem Einblick in partizipative Modelle im Handlungsfeld der Armutsprävention und -bekämpfung. Dies ist nötig, um Schein- oder Alibi-Partizipation zu vermeiden, und um einen Überblick in vielfältige Möglichkeiten der Mitwirkung zu erhalten. Anschliessend wird exemplarisch anhand von zwei Forschungsbeispielen (Begleitgruppe im BSV-Auftrag in der Schweiz und Evaluation im PartSoz-Projekt in Biel) auf Chancen und Voraussetzungen der Evaluation mit Einbezug von armutsbetroffenen und -gefährdeten Personen eingegangen. Abschliessend werden geeignete Evaluationsinstrumente und Indizien zur Messung des Wirkungspotenzials beschrieben. 


\section{Armut}

[4] Den meisten Menschen ist die finanzielle und materielle Dimension von Armut bekannt, das heisst, wenn ungenügend Geld beispielsweise für Wohnungsmiete oder Nahrungsmittel vorhanden ist. Armut hat noch weitere Dimensionen, die mit den ersten zwei Dimensionen und untereinander in Abhängigkeit stehen. Zu diesen zählen die soziale, kulturelle und gesundheitliche Dimension, wenn beispielsweise aufgrund von gesundheitlichen Einschränkungen oder fehlenden beruflichen Qualifikationen eine Berufsfindung stark eingeschränkt wird. ${ }^{1}$ In der modernen Gesellschaft ist Armut nach wie vor mit unterschiedlichen Vorurteilen, wie der Selbstverschuldung oder fehlender Anstrengung, behaftet, und ist damit ein stigmatisiertes Phänomen. Armutsbetroffene Personen erfahren einen Mangel an Selbstwirksamkeit und haben das Gefühl in der Gesellschaft keine Stimme zu haben. «Man verliert die Sprache, wenn man armutsbetroffen ist», erklärte eine betroffene Person. Solche verbreiteten Wahrnehmungen verstärken die soziale Isolation von Betroffenen und damit die Unsichtbarkeit von Armut in der Gesellschaft.

\section{Zentrale Dimensionen der Partizipation}

[5] In der Umsetzung von partizipativen Projekten und Massnahmen ergeben sich viele Gestaltungsspielräume, was die Tür zu Alibi- oder Schein-Partizipation öffnet und sowohl für Teilnehmende als auch für Projektleitende negativ ausfallen kann. Deshalb ist es wichtig, zentrale Dimension der Partizipation zu kennen und diese bei jedem Projekt zu definieren. Im Folgenden wird dies am Beispiel von Sozialdiensten skizziert. Detaillierte Ausführungen sind in CHIAPPARINI et al. ${ }^{2}$ nachzulesen:

[6] $\mathrm{Zu}$ definieren ist:

1. der Gegenstand, an dem betroffene Personen teilhaben können: in Sozialdiensten können armutsbetroffene Personen an der (Weiter-)Entwicklung von Strukturen und Prozessen von Sozialdiensten teilhaben;

2. der Zeithorizont und die strukturelle Einbettung der Partizipation: in Sozialdiensten können armutsbetroffene Personen befristet oder unbefristet an Gremien teilnehmen oder sind befristet oder unbefristet angestellt;

3. die Projektverantwortung: die Sozialdienste übernehmen diese selbst;

4. die Intensität der Partizipation: in Sozialdiensten können armutsbetroffene Personen ihre Meinung und Erfahrung mitteilen (Konsultation); sie können bei der Ausarbeitung von Entwicklungsaufträgen mitarbeiten (Co-Konstruktion), und in gewissen Projekten sind sie befugt, neben der Mitarbeit auch abschliessend mitzuentscheiden (Mitentscheidung); nur beim letztgenannten Intensitätsgrad von Partizipation wird die Entscheidungsmacht geteilt;

5. das übergeordnete Ziel der Partizipation: in den Sozialdiensten ist es das Ziel, dass armutsbetroffene Personen an der Optimierung von Organisationsstrukturen und -prozessen mitarbeiten; sie erarbeiten Verbesserungs-/Lösungsvorschläge und beurteilen professionelle

1 Emanuela Chiapparini/Claudia Schuwey/Michelle Beyeler/Caroline Reynaud/Sophie Guerry/Nathalie Blanchet/Barbara Lucas, Modelle der Partizipation armutsbetroffener und -gefährdeter Personen in der Armutsbekämpfung und -prävention, Forschungsbericht Nr. 7/20. Bundesamt für Sozialversicherungen (BSV), Bern 2020 (zit. Forschungsbericht BSV), S. 7.

2 Chiapparini/Schuwey/Beyeler/Reynaud/Guerry/Blanchet/Lucas, Forschungsbericht BSV, (Fn. 1), S. 10 ff. 
Praktiken; bei einer Anstellung ist es zudem möglich, dass betroffene Personen die Verbesserungs-/Lösungsvorschläge umsetzen;

6. die Staatsebene bzw. Verortung: die Projekte und Massnahmen, die im Sozialdienst vorhanden sind, verorten sich kommunal/lokal, kantonal/regional und/oder national.

\section{Partizipation in sechs Politik- und Handlungsbereichen der Armut- sprävention und -bekämpfung}

[7] Die Möglichkeit der Partizipation wurde im BSV-Forschungsprojekt ausgehend von den analysierten Projekten und Massnahmen in insgesamt sechs Handlungs- und Politikbereichen identifiziert, auf denen die folgenden Partizipationsmodelle basieren: ${ }^{3}$

- Evaluation und (Weiter-)Entwicklung von Strukturen und Prozessen von öffentlichen und privaten Dienstleistungsorganisationen: Sozialdienste organisieren befristete oder unbefristete Gremien, in denen Sozialhilfebeziehende Vorschläge zur Weiterentwicklung der Dienstleistungen einbringen können. (Modell 1)

- Ausbildung von Fachpersonen, die für die Implementierung von Armutspolitiken zuständig sind: Armutsbetroffene Personen arbeiten an der Entwicklung von Lehrplänen mit und beteiligen sich an der Ausbildung und an der Forschung. (Modell 2)

- (Weiter-)Entwicklung von politischen und rechtlichen Grundlagen: Staatliche Entscheidungsträgerinnen und -träger verschiedener Länder richten Gremien ein, in denen sich armutsbetroffene Menschen beteiligen, um politische und rechtliche Grundlagen im Bereich der Armutsbekämpfung zu erarbeiten oder zu optimieren. (Modell 3)

- Öffentliche/politische Diskurse: Betroffene Personen partizipieren an diesen beispielsweise mittels Beiträgen an Tagungen. (Modell 4)

- Gemeinschaftliche Selbsthilfestrukturen: In diesen unterstützen sich betroffene Personen mittels ihres Erfahrungswissens gegenseitig und stärken beispielsweise ihre Kommunikationsund Auftrittsfähigkeiten. (Modell 5)

- Erarbeitung von Grundlagen der Partizipation: Zu diesen Grundlagen gehört beispielsweise eine Charta mit Grundsätzen, an die sich alle Beteiligten während des Partizipationsprozesses zu halten haben. (Modell 6)

\section{Partizipative Evaluationsprojekte}

\section{Partizipative Evaluation}

[8] Parallel zur empowerment evaluation ${ }^{4}$ zielt der Ansatz der partizipativen Evaluation (PE) auf einen partnerschaftlichen Forschungsprozess zwischen den Evaluierenden und den Personen,

\footnotetext{
Chiapparini/Schuwey/Beyeler/Reynaud/Guerry/Blanchet/Lucas, Forschungsbericht BSV, (Fn. 1), S. 33 ff. David Fetterman/Abraham Wandersman, Empowerment Evaluation. In: American Journal of Evaluation 28 (2), Baltimore 2007, S. 179-198.
} 
welche die Befunde in die Praxis umsetzen. ${ }^{5}$ Hierbei steht nicht nur der Nutzen oder die Verbesserung des Projektes im Mittelpunkt, sondern auch der Anstoss von weiteren Entwicklungen oder das Ermöglichen von Lernprozessen.

[9] Weiter werden folgende Formen von PE unterschieden: ${ }^{6}$

1. Evaluatorgesteuerte PE: Die Evaluierenden treffen die relevanten Entscheidungen zur Evaluationsstudie.

2. Kollaborative PE: Die Evaluierenden und die primären Akteurinnen und Akteure treffen gemeinsam die Entscheidungen.

3. Akteursgesteuerte PE: Die Evaluierenden fungieren in einer Rolle als Dienstleister, die die Akteurinnen und Akteure in der Durchführung des Evaluationsprozesses befähigen und technische Unterstützung und Qualitätssicherung anbieten.

[10] Diese drei Typen sind an die Forschungstradition des user based reserach anschlussfähig, die folgende drei Formen unterscheidet: ${ }^{7}$

1. user involved research: Die betroffenen Personen haben eine Beratungsfunktion in der Forschung (Konsultation) inne, indem sie wichtige Informationen zu ihrem Alltag und ihrer Lebenserfahrung geben.

2. collaborative research oder partnership research: Die betroffenen Personen arbeiten partnerschaftlich im Forschungsprojekt oder in einzelnen Schritten von diesem mit (Mitentscheidung).

3. user-controlled research oder user-lead research: Die betroffenen Personen führen selbstständig Forschungsprojekte gemäss gängigen Forschungsstandards durch (Selbstverwaltung).

[11] In den folgenden Kapiteln werden diese drei Formen exemplarisch veranschaulicht.

\section{Begleitgruppe als kollaborative partizipative Evaluation}

[12] Das BSV-Forschungsprojekt ${ }^{8}$ hatte eine Begleitgruppe, in der zwei armutsbetroffene Personen, fünf Interessensgruppen-Vertretende und drei Fachpersonen des BSV beteiligt waren. Der Begleitgruppe kam eine evaluierende Funktion zu, die der kollaborativen partizipativen Evaluation zugeordnet werden kann. So beurteilten alle Mitglieder der Gruppe die eingegangenen Offerten. Weiter diskutierten und beurteilten sie den Forschungsprozess in drei Sitzungen (Themen: Kategoriensystem, Zwischenbericht, Schlussbericht) und in einem Workshop (Thema: Auswahl

5 Sven Brandes/Ina Schaefer, Partizipative Evaluation in Praxisprojekten, In: Praev Gesundheitsf 8 (3), Bielefeld 2013, S. 134.

6 Brandes/Schaefer, Partizipative Evaluation in Praxisprojekten, (Fn. 5), S. 133.

7 Emanuela Chiapparini/Véronique Eicher, User Involvement in der Sozialen Arbeit - Anknüpfungspunkte für Praxis-, Forschungs- und Lehrprojekte in der Schweiz. In: Schweizerische Zeitschrift für Soziale Arbeit 24.18, Zürich/Genf 2019, S. 117-134.

Weiterführende Literatur: Peter Beresford, From «other» to involved: user involvement in research: an emerging paradigm. In: Nordic Social Work Research 3 (2), Maastricht 2013, S. 139-148.

8 Chiapparini/Schuwey/Beyeler/Reynaud/Guerry/Blanchet/Lucas, Forschungsbericht BSV, (Fn. 1). 
der Vertiefungsmodelle). Alle Teilnehmenden der Begleitgruppe erhielten die gleiche Spesenentschädigung. Zudem stand den armutsbetroffenen Personen die Möglichkeit einer Vorbereitung auf die Sitzung zur Verfügung, um die Besprechungsthemen zusammen mit einer Fachperson durchzugehen und vorzubesprechen. Diese Möglichkeit wurde genutzt und als nützlich empfunden. Zudem brachten beide armutsbetroffenen Personen Erfahrungen in andere Partizipationsprozesse und Interessensorganisationen ein.

\section{Indikatorengewinnung durch evaluatorgesteuerte partizipative Evaluation (PartSoz)}

[13] Im laufenden Projekt «Partizipative Sozialhilfe» (PartSoz) wird ein partizipatives Austauschgefäss «Gemeinsam/Ensemble» mit dem Ziel explorativ evaluiert, geeignete partizipative Erhebungsinstrumente und Indikatoren für Wirkungsmodelle zu gewinnen. Das Austauschgefäss «Gemeinsam/Ensemble» setzt sich aus sieben sozialhilfebeziehenden Personen, drei Fachpersonen der sozialen Arbeit und einem externen Moderator zusammen. Eine Durchführung des Austauschgefässes umfasst fünf Sitzungen und wurde seit 2019 zwei Mal durchgeführt. ${ }^{9}$ In den ersten drei Sitzungen werden Ideen, Vorschläge und Anliegen zu einem breiten Thema diskutiert und konkrete Vorschläge erarbeitet (z. B. war das Thema der ersten Durchführung «Der Eingang der Sozialhilfe» und das Thema der zweiten Durchführung «Partizipationsmöglichkeiten in der Sozialhilfe»). In der vierten Sitzung stellen die armutsbetroffenen Personen ihre Vorschläge der Leitung Sozialhilfe vor. Diese prüft die Vorschläge auf die Umsetzbarkeit und gibt in der fünften Sitzung, etwa einen Monat später, eine Rückmeldung, welche Vorschläge wann und wie umgesetzt werden und was aufgeschoben wird oder nicht umsetzbar ist.

[14] Innerhalb des Austauschgesprächs finden sich ebenfalls akteursgesteuerte partizipative Evaluationsmomente: Durch die Rückfragen des Moderators nach jedem Arbeitsschritt und nach jeder Sitzung wird der Konsens des Arbeitsprozesses sichergestellt und den Teilnehmenden die Möglichkeit gegeben, Themen, Arbeitsgeschwindigkeit und eigene inhaltliche Schwerpunkte zu setzen. Weiter bestimmen die Teilnehmenden die Relevanz der selbst eingebrachten Anliegen, Ideen und Vorschläge kollektiv. Schliesslich beurteilen die Teilnehmenden in einer Schlussevaluation basierend auf graphischen Darstellungen das Austauschgefäss kollektiv.

\section{Chancen und Voraussetzungen ${ }^{10}$}

[15] Als besonderer Mehrwert der partizipativen Evaluation stehen die unmittelbaren Wahrnehmungen und Beurteilungen der betroffenen Personen im Zentrum. Durch die Perspektive der betroffenen Personen konnte aufblinde Punkte hingewiesen werden, worüber die Fachpersonen selbst

9 Emilie Clavel, Die Betroffenen beeinflussen die Prioritäten des Managements, Zeitschrift für Sozialhilfe (ZESO) (2), Zürich 2020.

10 Die folgenden Ausführungen basieren auf unterschiedlichem Quellenmaterial: Erstens auf Rückmeldungen der Begleitgruppe während des Forschungsprozesses und in den Gremiumssitzungen, die verschriftlicht wurden. Zweitens auf recherchierten Wirkungspotentialen und Befunden zum Partizipationsmodell 1 (vgl. CHIAPPARINI/ Schuwey/Beyeler/Reynaud/Guerry/Blanchet/Lucas, Forschungsbericht BSV, (Fn. 1). Drittens auf einem ausführlichen leitfadengestützten Interview mit einer Teilnehmerin der Begleitgruppe und schliesslich auf erste Befunde aus dem PartSoz-Projekt. 
überrascht waren oder die sie in dieser unmittelbaren Weise nicht gesehen und aufgenommen hätten. Eine Fachperson für Soziale Arbeit formuliert dies exemplarisch wie folgt: «Die Sozialhilfeempfangenden erweiterten mir den Blick - durch gemeinsamen Austausch sind wir auf Ideen gekommen, auf die ich mit meinen Kolleginnen alleine nicht gekommen wäre». Ebenso wiesen einzelne Sozialhilfeempfangende eine anfängliche grundsätzliche Abwehrhaltung gegenüber den Fachpersonen für Soziale Arbeit auf, die sich durch die Zusammenarbeit bzw. die partizipative Evaluation des Eingangsbereichs des Sozialdienstes in eine Akzeptanz wandelte, was zu einer Ablegung von Missverständnissen führte. So konnte beispielsweise geklärt werden, dass eine Sicherheitsperson der Securitas beim Eingang des Sozialdienstes zum Schutz der Sozialhilfeempfangenden, und nicht zu ihrer Einschüchterung, dort steht.

[16] Zudem haben die getroffenen Entscheidungen und die erzielten Resultate durch die gemeinsame Evaluation erheblich an Legitimation und Glaubwürdigkeit gewonnen.

[17] Das Vertrauen der betroffenen Personen zu gewinnen ist wichtig, damit sie sich trauen, auch kritische Äusserungen zu ihrem Verhalten und zum Projekt zu machen. Auch das Finden einer Balance zwischen Steuerung der Evaluierenden und Selbstbestimmung der betroffenen Personen stellte sich als herausfordernde, schwierige Aufgabe heraus. Um diese Herausforderungen anzugehen und einen partizipativen Prozess zu ermöglichen, sind unterschiedliche Voraussetzungen nötig.

- Primär ist eine sorgfältige Planung sicherzustellen: Konkrete und erreichbare Ziele, die jeweiligen Rollen, Befugnisse und die Spesenregelung sind, wenn möglich, zusammen mit den armutsbetroffenen Personen festzulegen. Die festgelegte und kommunizierte Organisation des Arbeitsprozesses ermöglicht, dass jede Person weiss, was sie zu tun hat und wo die Handlungsgrenzen sind. Dies ist wichtig, weil manchmal die Meinung vorherrscht: «Wenn Betroffene noch mitmischen, gibt es gar kein Resultat mehr», oder «Betroffene erhalten plötzlich eine zu starke Stimme ».

- Besonders motivierend und zielführend sind Partizipationsprozesse, in denen eine «Machtverschiebung» ermöglicht wird und armutsbetroffene Personen im Entscheidungsprozess mitwirken oder zumindest informiert werden, welche der erarbeiteten Vorschläge wie und wo umgesetzt werden.

- Durch Nachfragen von Fachpersonen oder eines externen Moderators können Kompetenzen und Potenziale der betroffenen Personen aktiviert und genutzt werden. Betroffene erleben sich mit ihren persönlichen Armutserfahrungen als Expertinnen und Experten, und Vorurteile wie das der «faulen und dummen» Armutsbetroffenen können abgebaut werden. Aktive Beiträge von armutsbetroffenen Personen ermöglichen, dass alle beteiligten Personen erfahren, dass armutsbetroffene Personen in der Gesellschaft etwas Nützliches und Aufbauendes leisten können.

- Ein bewusstes und selbstreflexives Verhalten bei den Fachpersonen ist eine zentrale Voraussetzung, damit Partizipation nicht als Alibiübung verstanden wird. Hierzu gehören etwa angemessene Umgangsformen wie die Begegnung «auf Augenhöhe», insbesondere bei bestehenden Hierarchien aufgrund beruflicher Funktionen oder Kommunikationsfertigkeiten.

- Von Vorteil ist, wenn die armutsbetroffenen Personen wie auch die Fachpersonen an Partizipationsprozessen bereits teilgenommen haben oder über Kommunikationsfähigkeiten verfügen. Es geht darum, eine gewisse Distanz zur eigenen Biografie oder Erfahrung zu schaffen, oder die «Expertenrolle» abzulegen und eine lernende Haltung einzunehmen. 
- Es bewährte sich, dass armutsbetroffene Personen und Fachpersonen ein Schulungs- oder Vorbereitungsangebot erhielten, um sich auf die Sitzung vorzubereiten und die eigenen Haltungen zu reflektieren.

\section{Geeignete Evaluationsinstrumente und Indizien für die Messung von Wirkungspotenzial}

[18] Das BSV-Forschungsprojekt zeigte ebenfalls eine ungenügende Erforschung der Wirkung von Partizipationsprozessen in Massnahmen und Prozessen der Armutsprävention in der Schweiz und international auf. ${ }^{11}$

[19] Als geeignete Evaluationsinstrumente zeichneten sich im Forschungsprojekt PartSoz die Folgenden ab:

- Leitfadengestützte Einzelinterviews, mit Möglichkeiten, eigene Themen und Schwerpunkte zu setzen. Diese eigenen sich auch, um Evaluationskriterien zu gewinnen.

- Gruppeninterviews, in denen sie sich nicht als Einzelne zu exponieren haben und mit denen eine kollektive Meinung gewonnen wird.

- Grafische Evaluationen und Visualisierung von getroffenen Entscheiden in der Arbeitsgruppe.

- Ethnografische Beobachtungen eignen sich ebenfalls, wobei hierzu der Forschungszugang herausfordernd ist; denn bei den Leitenden besteht die Meinung, dass die Gruppendynamik beeinflusst wird. Deshalb wird empfohlen, dass die Forschenden eine offizielle Funktion im Gremium einnehmen und das schriftliche Einverständnis der Teilnehmenden einholen. Im Arbeitsgremium des BSV-Projekts «Partizipation armutsbetroffener und -gefährdeter Personen - Praxishilfen» organisierten die Forschenden das Gremium so, dass die ethnografische Beobachtung durch die protokollierende Person durchgeführt wurde. Dies stiess sowohl bei den Auftraggebenden als auch bei den Beteiligten des Projekts auf allgemeine Akzeptanz.

[20] Schreiben ist für die Teilnehmenden nicht eine alltägliche Tätigkeit, sodass der Einsatz von Fragebögen im PartSoz-Projekt nach Rücksprache mit den betroffenen Personen als unpassend eingeschätzt wurde.

[21] Zudem eignen sich Einzelinterviews und Gruppendiskussionen besonders, um Evaluationskriterien und Indizien für das Wirkungspotenzial zu gewinnen.

[22] Indizien für das Wirkungspotenzial gehen beispielsweise aus folgenden Aussagen einer armutsbetroffenen Person hervor (was im Folgenden von einer Person auf den Punkt gebracht wird, erhärtet sich durch ähnliche Aussagen aus anderen Interviews):

[23] «Ich habe in der Begleitgruppe gespürt, dass wir [die teilnehmenden betroffenen Personen] akzeptiert worden sind, zum Beispiel, wenn wir sagten, ihr müsst eine einfache Sprache nehmen, sonst versteht es niemand». Oder: «Wir hatten in dieser Gruppe das Gefühl, dass wir ernst genommen wurden - man hörte uns zu. Und das hat viel gebracht.»

[24] Aus solchen Textpassagen verdichteten sich im PartSoz-Projekt folgende Indizien für das Wirkungspotenzial, wobei das Folgende nicht als abschliessende Auflistung zu verstehen ist:

11 Chiapparini/Schuwey/Beyeler/Reynaud/Guerry/Blanchet/Lucas, Forschungsbericht BSV, (Fn. 1). 
- Gefühl und Wahrnehmung, Erfahrungs- und Fachwissen einbringen zu können

- Gefühl und Wahrnehmung, «auf Augenhöhe» zu sein

- laufende Überprüfung dieser Wahrnehmung

- verständliche und offene Kommunikation über die Organisation des Arbeitsprozesses

- Definition und Kommunikation der Verantwortlichkeiten, der erreichbaren Ziele, der jeweiligen Rollen, Befugnisse und der Spesenregelung

- laufende Rückfragen, um allfälligen Missverständnissen vorzeitig vorzubeugen.

[25] Anhand solcher und weiterer Indizien für das Wirkungspotenzial der Partizipation von armutsgefährdeten und -betroffenen Personen können relevante partizipative Evaluations- und Forschungsprojekte vermehrt auf Basis von sozialwissenschaftlichen Methoden geprüft werden. Denn nur dadurch kann der Wissensstand über die Umsetzung von partizipativen Evaluationen erhöht, und können Wirkungen und Verbesserungspotenziale der partizipativen Forschungsund Praxisprojekte erfasst und aufgezeigt werden.

Emanuela Chiapparini, Prof. Dr., ist Dozentin in Sozialer Arbeit mit Schwerpunkt Partizipationsforschung in Schule, Freizeit und Armutspolitik an der Berner Fachhochschule, E-Mail: emanuela.chiapparini@bfh.ch. 